\title{
Development and Evaluation of a Novel Interprofessional Learning Activity Addressing the Management of Phenylketonuria
}

\author{
Heather Donald, Morag C. E. McFadyen, Susanne P. Boyle* \\ School of Pharmacy and Life Sciences, Faculty of Health and Social Care, Robert Gordon University, \\ Aberdeen, UK \\ Email: *S.boyle@rgu.ac.uk
}

Received January 20 ${ }^{\text {th }}$, 2013; revised February $24^{\text {th }}, 2013$; accepted March $7^{\text {th }}, 2013$

\begin{abstract}
This new initiative across the School of Pharmacy and Life Sciences at Robert Gordon University focuses around students from the Overseas Pharmacist's Assessment Programme (OSPAP) and 3rd year Nutrition and Dietetics students working in an interprofessional setting to manage the care of individuals with Phenylketonuria (PKU). A problem based learning approach was employed which involved small group interprofessional working to solve a series of simulated case studies concerned with the management of phenylketonuria. The session was further enhanced by an expert patient narrative and the opportunity to test a series of commercially available low protein/low phenylalanine food substitutes provided by SHS-Nutricia and Vitaflo which are used in the disease management. Group evaluation of the activity was generally positive with all groups indicating that the learning objectives had been achieved in a setting which promoted collaborative interprofessional working, acquisition of knowledge pertaining to the management of PKU and a format which enabled a breadth and depth of material to be covered in a relatively short time. The contributions of the expert patient and availability of test samples enhanced the interactivity of the session and provided a meaningful insight into the real life scenario of PKU patients. Limitations of the session included the rather narrow range of healthcare professional students involved in the activity and the need for better signposting of preparatory reading material. Planned future developments of this initiative aim to involve health visiting, biomedical science and social work students to enable a more holistic approach to the health and social care issues of PKU to be considered and the inclusion of an Articulate quiz activity for student self evaluation pre event. In conclusion this initiative addressed the 6 learning objectives in an interactive manner which received positive learner feedback and the academic team has identified a clear strategy for future development.
\end{abstract}

Keywords: Interprofessional Learning; Phenylketonuria; Nutritionists; Dietitians; Pharmacists; Problem-Based Learning

\section{Interprofessional Education in Pre-Registration Courses}

This study describes the rationale, development and evaluation of a novel interprofessional (IPE) learning experience involving postgraduate non EEA qualified pharmacists enrolled as OSPAP students and senior undergraduate Nutrition and Dietetics students.

The OSPAP students are engaged in full-time Master's level study on a programme accredited by the General Pharmaceutical Council for non EEA qualified pharmacists who wish to retrain and apply for entry to the UK pharmacy pre-registration training scheme. They tend to be a very heterogeneous student population in terms of age, ethnicity (see Figure 1) and professional experience. The two key commonalities they share are an English language proficiency of IELTS band 7 or greater and a BPharm degree (or equivalent) which entitles them to register as a pharmacist in their home country. The Nutrition and Dietetics (N \& D) students on the other hand are a homogeneous group in terms of age and ethnicity.

The IPE event builds upon existing IPE activities within the Faculty of Health and Social Care, Robert Gordon University (Robertson \& Joseph, 2012) and the development required the

\footnotetext{
*Corresponding author.
}

collaborative activities of two Faculty IPE Champions and the OSPAP Programme Leader. Whilst N \& D students participate in Faculty IPE events from years 1 - 4 (see Figure 2) the shorter duration (9 - 12 months) of the OSPAP courses necessitates a more bespoke approach and this initiative was developed to partly address that need.

Preliminary discussions identified an area of common teaching across the curricula (Barr \& Low, 2012), namely Phenylketonuria, which was being addressed by a rather didactic lecture format. It was believed that this metabolic condition could form the basis of a meaningful interprofessional learning event which if properly planned and effectively facilitated had the potential to address the educational standards defined by the General Pharmaceutical Council (GPhC) and the Health Care Professions Council (HCPC) for pharmacy and healthcare professions students respectively. Specifically the event sought to provide OSPAP students "practical experience of working with patients, carers and other healthcare professionals" and "learning based on experience that provides education in interprofessional practices and procedures with other healthcare professionals”, (GPhC, 2011) and Nutrition and Dietetics students an activity within which "the profession-specific skills and knowledge of each professional group” would be adequately addressed (HCPC, 2013). 


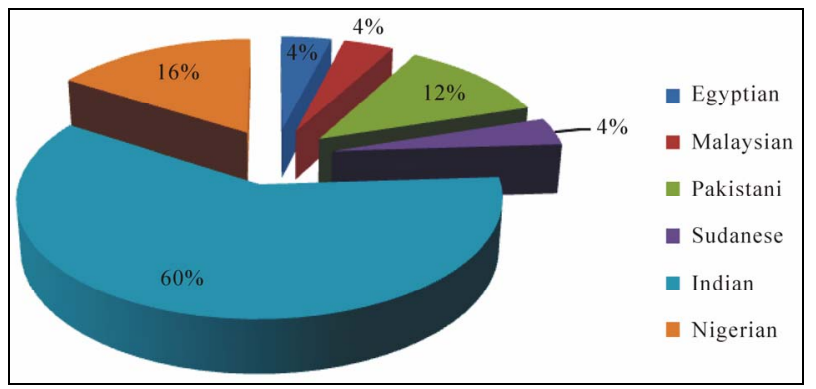

Figure 1.

Ethnicity of OSPAP population ( $\mathrm{n}=25$ postgraduate students).
Phenylketonuria is an error of protein metabolism which is screened for at birth in the United Kingdom (UK) and primarily managed through diet with the use of prescription products (e.g. low protein and phenylalanine free products, NSPKU, 2010a) and in selected cases by use of $\operatorname{Kuvan}^{\circledR}$, a prescription medication licensed for use in hyperphenylalinemia (EMEA, 2011; Horsley, 2009; NSPKU, 2010b). Clearly nutrition and dietetics professionals have a pivotal role to play in PKU management however it is recognised that PKU is most effectively managed through a multi-disciplinary team approach (NSPKU, 2004) and that pharmacists have a contribution to make in ensuring the correct dispensing of prescription food substitute products, by liaising with prescribers and dieticians if medicines contain

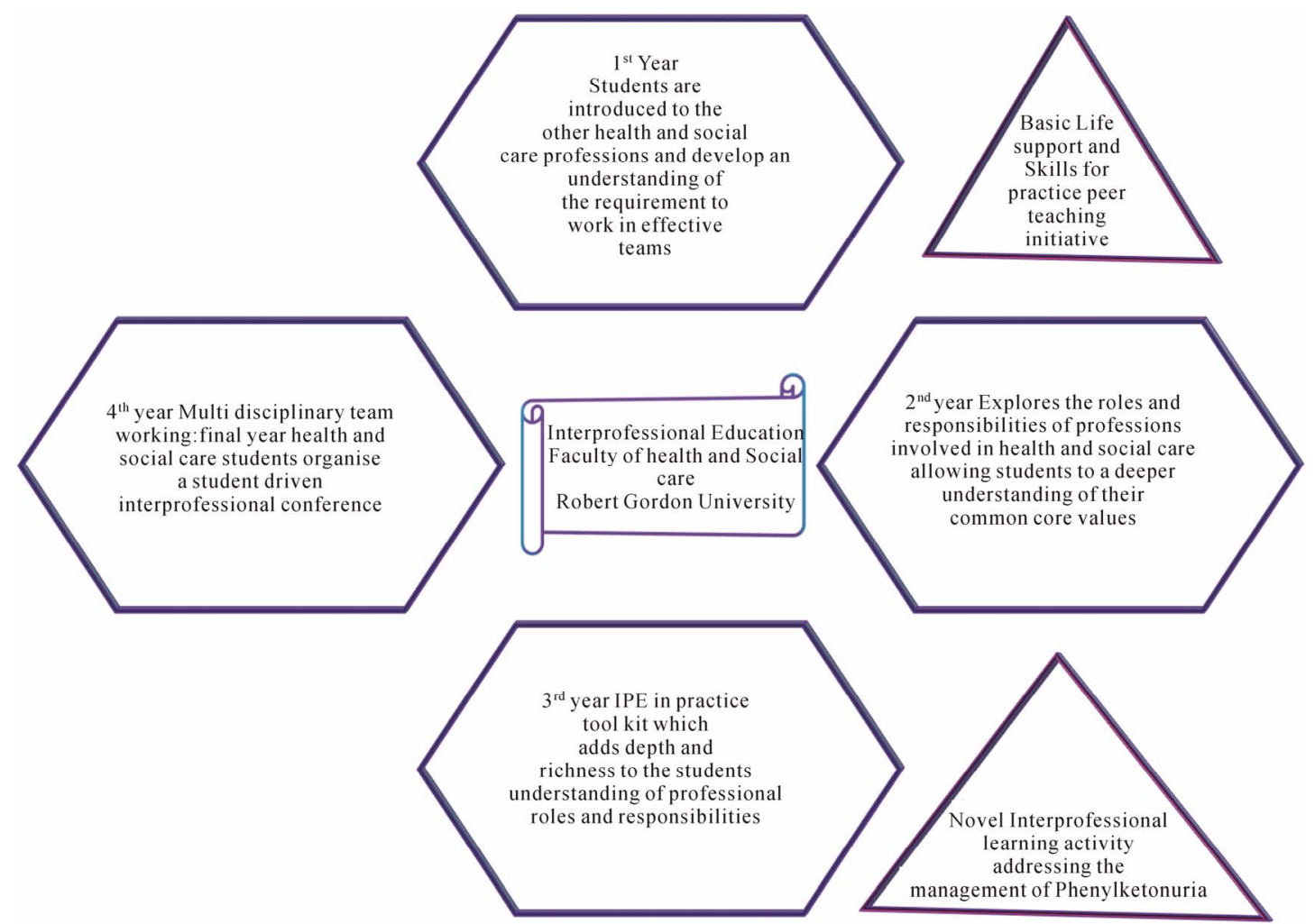

Figure 2.

Schematic representation of interprofessional activities within the Faculty of Health and Social Care.

aspartame or phenylalanine, by providing advice on prescription charges (White \& Farooq, 2009) and in the management of $\operatorname{Kuvan}^{\circledR}$ treated patients (Horsley, 2009).

\section{Design and Delivery of the IPE Activity}

A blended learning approach was adopted with preparatory reading material including overview lecture slides; key guidelines for PKU management, a model for reflective learning (Gibbs, 1988); details of student groupings and the key learning objectives (see Table 1) being provided in advance of the session via the University's virtual learning environment.

A total of 51 students (24 OSPAP and 27 nutrition and dietetics students) were assigned to 9 mixed healthcare professsional groups. The first 30 minutes was assigned to an ice breaker activity with student groups being encouraged to informally share their backgrounds and personal insight to PKU.
The primary IPE activity took the form of a problem-based learning approach (Thompson, 2010) with individual groups being tasked with identifying solutions to a series of unseen case studies $(n=6)$ concerned with the management of phenylketonuria (PKU). The IPE event was enhanced by the contribution and reflections of: a) an expert PKU patient narrative and b) by the inclusion of two commercially sponsored stands (SHS-Nutricia and Vitaflo UK) containing promotional literature and a wide range of low protein and low phenylalanine substitute products which students were encouraged to taste.

The sessions was facilitated by 3 academics, 51 students participated in the event and student groups were allocated 90 minutes $(2 \times 45$ minute periods) to complete and feedback their thoughts and conclusions on the management issues presented in two of the 6 case studies. The expert patient discussed her personal experiences of living with PKU with each of the 9 
Table 1.

The key learning objectives of the IPE activity.

Develop effective communication skills and work collaboratively with other healthcare professional students to solve a range of case studies concerned with management of phenylketonuria.

Describe phenylalanine metabolism in phenylketonuria (PKU).

Describe the cause of classic PKU and the role of tetrahydrobiopterin in phenylalanine metabolism.

Summarise the blood phenylalanine levels associated with the 3 different phenotypes; classic PKU; variant PKU and mild hyperphenylalinemia (HPA).

Demonstrate knowledge on dietary restrictions for PKU.

Describe appropriate strategies for PKU clinical management, including diet and treatment.

Table 2.

Group evaluations of interprofessional working.

\begin{tabular}{cc}
\hline Group identifier & Challenges of mixed group working \& resolution identified \\
A & "We found it really easy to work together as a group of professionals" \\
B & "No-we worked well together as a group" \\
C & "It was easy to interact with each other because the given case studies were mainly focussed on basics of each profes- \\
sion which were quite easy to understand" \\
D \\
E - G \\
H \\
I
\end{tabular}

groups and additionally all groups had the opportunity to consult with the company representatives and discuss and sample the various food substitute products available.

\section{Evaluation of Effectiveness and Impact upon Learners}

At the end of the event each group was asked to anonymously complete a brief evaluation form which addressed 4 key factors namely: 1) the extent to which the group perceived the learning objectives had been achieved; 2) any issues experienced with mixed group workings and the resolutions identified; 3) the strengths of the IPE session; and 4) the weaknesses of the IPE session.

The students were also asked to submit a brief reflective piece (100 - 500 words) within 10 days of the event with participation and completion of the reflective writing leading to individual learners receiving a certificate of completion.

All 9 groups (designated A-I for reporting) completed and returned the event evaluation form and the feedback was generally positive. Student groups were asked to score how well they feel they had achieved the session learning objectives with a score of 5 being "completely achieved" and a score of 1 being "not achieved". The mean score was 4.6 and only one group (Group D) recorded any notable difficulties with the mixed group working whilst the majority reported "no challenges" or found it "easy to work together", see Table 2.

Strengths of the session were identified by all 9 groups and included positive comments (see exemplar quotes right) regarding the case study problem-based learning approach, the opportunity to learn about other health professional roles and the opportunity to share knowledge, skills and experiences in a more informal setting than lectures afford.

"Working together with other healthcare professional promotes interdependence; opportunity to share knowledge, skills, experience; much more informal than lectures; a lot covered within a short period including case studie".

"Pharmacists and dieticians working together can easily address dietary and clinical requirements of patient. This session provided very good experience about how this goal can be achieved in real life scenarios".

"Both professionals respected each other's viewpoints, this made communication good and effective-very good approach to IPE \& learning, well run and beneficial”.

"It was such an informal and relaxed atmosphere and I was amazed at how much I was able to learn within such a short period of time".

"It was an eye opening session for me as I had never come across a case of PKU in my work as a pharmacist in my own country. This is due to the fact that PKU is more common in Caucasians than in Asians".

"A PKU patient was presented during the session and we had a chance to chat with her and it helped a lot in understanding real life scenario of the disease."

"I feel I have learned more about PKU for learning inter-professionally than I would have if I had just received a lecture on it. This type of learning really make me think and it was great to meet new people".

"I think it was an excellent learning experience. I thought that the OSPAP students were interested to learn for us and vice versa. It was a very practical learning set- 
ting and the case studies were interesting".

"Positive experiences from the IPE event were team working with another professional group. I learnt the role of a pharmacist and having a patient present what it was like living with the condition helped our learning".

"Overall the session for me went really well because I felt I learnt a lot from working as a group and from the OSPAP students. We were able to complete our case studies quite easily because we all contributed and everyone took on board what everyone else was saying. The OSPAP students were really good at working out the dosages and they took the time to explain to us how they worked it out, which was really interesting for us".

"Speaking to the student with PKU was very interesting and helped us understand what it can be like to live with PKU”.

"I enjoyed this interprofessional way of learning immensely and found that this method of learning together extremely helpful. I got on well with the group that I was in and I felt able to contribute to the discussion as well as learn from the others. Overall, I think there was a great outcome from this event and I felt really happy that I attended and got to know new people from another profession as well as learn about PKU".

The weaknesses identified by 5 groups included a lack of audibility within the venue during the group feedback on the case studies; a desire for a wider range of healthcare professional students to be involved in the event and the perception that the roles of nutrition and dietetic students enabled them to contribute more to the case studies. However this viewpoint was balanced by a group view that "Kuvan-the only available drug to treat PKU was not addressed properly".

A total of 29 students submitted the reflective exercise and once more the impact of the session was evident in many student submissions:

"I was continuously thinking in the exercise about what I would do in given scenario as a pharmacist. At the end of the exercise I became clear about the place of dietician in patient care and feeling more confident about requesting or referring dietician's services".

\section{Discussion}

This study summarises a new IPE initiative which is novel in both its interactive format and in the endeavours to bring together a disparate group of health professional students (see Figure 1), many with international professional training backgrounds to work collaboratively on a metabolic disease. Both national and international drivers for IPE exist with the World Health Organisation (WHO) providing a framework for action on IPE and collaborative practice which notes "Interprofessional education occurs when two or more professions learn about, from and with each other to enable effective collaboration and improve health outcomes”, WHO (2010: p. 13). National drivers include the educational standards set by regulatory bodies in the UK namely the General Pharmaceutical Council (GPhC, 2011) concerned with OSPAP course accreditation and pharmacy pre-registration training and the Health Care Professions Council (2009).

The development of this activity required significant collaborative working by 3 academics and the facilitation of the event required a staff student ratio of 1:17 higher than that normally required for delivery of the existing PKU sessions within the curricula. It is clear however that the student learners perceived a number of benefits from the new format and that the 6 learning objectives had been achieved. Critically student feedback showed a measure of insight onto the strengths of IPE which was elegantly reflected in the feedback of one group who noted "Working together with other healthcare professional promotes interdependence; opportunity to share knowledge, skills, experience; much more informal than lectures; a lot covered within a short period including case studies".

In addition to planning for the differences in academic stage the development team was mindful of possible cultural differences in approaches to diagnosing or managing PKU in other continents. To that end one of the case studies specifically explored learners knowledge of how PKU was managed in a range of countries outwith Europe. The reality however was that few, if any, of the student learners had any real personal or professional experience of PKU and this perhaps reflects the relatively low disease incidence (1 in 10,000 births) in European populations with lower incidences being reported in sub-Saharan populations (Donlon et al., 2004). Notwithstanding this low incidence the management of PKU in the UK requires an effective national screening programme, achieved through a multi-disciplinary team approach (NSPKU, 2004) and affected individuals are expected to commit to lifetime compliance on a restricted protein/phenylalanine diet. It is imperative that both healthcare professions appreciate the impact of such disease and the contributions they may make individually and via collaborative professional working to ensure effective lifetime management of the condition. In that respect this goal has also been achieved with group feedback noting that the session both increased knowledge of PKU and exemplified how pharmacists and dieticians may work together to address dietary and clinical needs of PKU affected individuals.

Gibbs’ Reflective Cycle (Gibbs, 1988) builds upon Kolb’s experiential learning cycle (Kolb, 1984) and proposes that theory and practice enrich each other in a never-ending circle. Kolb's experiential learning cycle places reflection in a central position pedagogically (Kolb, 1984). In this model students' learn more effectively if they are actively involved in the learning process than if they are passive receivers and this outcome was echoed in the thematic analysis of the students' reflection. Similarly it was appreciated that the experiences of patients is a valuable way to inform individual appreciation and professional attitudes towards conditions such as PKU and the session was enhanced significantly by the narrative of a young adult PKU sufferer who was also completing the nutrition and dietetics degree programme. Student feedback on this element of the session was again generally positive and a number of student reflections noted the merit of this element echoed in one student view: "It was also worthwhile to speak to someone who had the condition. This helped me to understand the treatment from a patient's viewpoint".

Post session delivery and review of student evaluations the academic development team also reflected upon the pros and cons of the event (design and content) and 3 key areas for future academic development have been agreed. These include the expansion of the range of healthcare profession students participating in the event in parallel with a development of the existing cases studies to provide a more holistic health and social care approach. Ideally we are seeking to involve health 
visiting students, biomedical science students (NSPKU, 2004) and social work students (NSPKU, 2004) enabling both the biochemistry of blood monitoring and issues pertaining to heath and welfare to be more meaningfully addressed. Secondly it is believed that a rationalisation of the range of resources provided as preparatory activities needs to be undertaken and the blended learning approach be developed to include a measure of self evaluation pre session e.g. a formative Articulate quiz activity. Thirdly the facilitators note the concerns expressed regarding the perceived imbalance in dietetic and prescribing issues associated with each case study and it has been agreed that selected case studies will be modified to address the issues of medication substitutes to avoid the use of aspartame containing formulations (White \& Farooq, 2009).

Finally on a practical note and in response to group feedback that audibility of the narration during case study conclusions was compromised, largely by the desire of other groups wishing to discuss their own associated findings, we plan to revise the format of this element. Thus whilst such learner discussion is laudable it did detract from the experience of some students and so it is agreed that the feedback session adopt a more formal platform with student groups providing a brief 4 - 6 minutes summary of their key findings supported by reference to flipchart annotations and a further 2 - 3 minutes will be allocated for questions.

\section{Conclusion}

In conclusion learner feedback suggests that this initiative has embraced the ethos of IPE and enabled future UK healthcare practitioners to experience a "quality education that enable inter-professional learning in classroom and practice contexts", CAIPE (2009). Future development of this activity will seek to increase the number of healthcare professional students contributing to the shared learning, to focus the preparatory activities by linking personal study to individual self evaluation by means of an Articulate quiz and to continue to develop the case studies to encourage pharmacy students to consider other issues of aspartame use in both prescription and over the counter medications.

\section{Acknowledgements}

The authors wish to acknowledge both the healthcare professional students who contributed fully and professionally to this event and its associated evaluation and the contributions of the expert patient and the SHS Nutricia and Vitaflo UK company representatives who enhanced the content and delivery of this new IPE development.

\section{REFERENCES}

Barr, H., \& Low, W. (2012). CAIPE interprofessional education in preregistration courses. A caipe guide for commissioners and regulators of education. URL (last checked 3 January 2013). http://www.caipe.org

Donlon, J., Levy, H., \& Scriver, C. R. (2004). Hyperphenylalaninemia: Phenylalanine hydroxylase deficiency. In Scriver, C. R. et al., (Eds.) The metabolic and molecular bases of inherited disease. New York: McGraw-Hill.

European Medicines Agency (EMA) (2011). European public assessment report for Kuvan (EMA/173967/2011). URL (last checked 4 January 2013). http://www.ema.europa.eu/Findmedicine/Human medicines/European

General Pharmaceutical Council (GPhC) (2011).Standards for the education and training of non-EEA pharmacists wanting to register in Great Britain. URL (last checked 3 January 2013).

http://www.pharmacyregulation.org/sites/default/files/Standards\%20 for\%20the $\% 20$ education $\% 20$ and $\% 20$ training\%20of\%20non-EEA\%2 0pharmacists\%20wanting\%20to\%20register\%20in\%20GB.pdf

Gibbs, G. (1988). Learning by doing: A guide to teaching and learning methods. Oxford: Oxford Brookes University Further Education Unit.

Healthcare and Professions Council (HCPC) (2009). Standards of education and training (SETs) cross-mapping document. URL (last checked 3 January 2013).

http://www.hpc-uk.org/search/index.asp?action=search\&sQuery=inte rprofessional+learning

Horsley, Wm. (2009). Sapropterin (Kuvan ${ }^{\circledR}$ ) in the management of phenylketonuria. NHS North East Treatment Advisory Group, 1-18.

Kolb, D. A. (1984) Experiential learning: Experience as the source of learning and development. Englewood Cliffs, NJ: Prentice Hall.

NSPKU (2004). Management of PKU. A consensus document for the diagnosis and management of children, adolescents and adults with Phenylketonuria (pp. 1-18). The National Society for Phenylketonuria (United Kingdom) Ltd. http://www.nspku.org

NSPKU (2010a). The prescription of low protein foods in PKU. The National Society for Phenylketonuria (United Kingdom) Ltd. http://www.nspku.org

NSPKU (2010b). The role of Sapropterin (Kuvan) in the management of PKU in the NHS: Considerations and prospects. The National Society for Phenylketonuria (United Kingdom) Ltd. http://www.nspku.org

Robertson, J., \& Joseph, S. (2012). Interprofessional education for biomedical students: The Aberdeen experience. The Biomedical Scientist, 56, 492-496.

Thomson, C. (2010). Do inter-professional education and problem-based learning work together? The Clinical Teacher, 7, 197-201.

White, F., \& Farooq, S. (2009). The pharmacist's role in the management of Phenylketonuria. Hospital Pharmacy Europe, 45, 60-61.

WHO (2010). Framework for action on interprofessional education and collaborative practice. Geneva: World Health Organisation. 